ISSN 2693-2458

\title{
Comparison of Sevelamer and Calcium Carbonate on Lipid Profile \& Hba1c in Diabetic Kidney Disease
}

\author{
Journal of Diabetes and Endocrinology Research
}

Research Article

Jais Kumar*, Naveed Sarwar, Muzaffar Shoaib and Syed Ali Naqi

Department of Nephrology, Akbar Niazi teaching Hospital, IMDC, Pakistan

\author{
*Correspondence author \\ Jais Kumar \\ Department of Nephrology \\ Akbar Niazi teaching Hospital \\ IMDC \\ Pakistan
}

Submitted : 6 Oct 2020 ; Published : 20 Nov 2020

\begin{abstract}
Diabetic is a worldwide health issue and diabetic kidney disease is a dreaded complication of the disease. Drugs that can impair intestinal absorption of advanced glycation end products (AGE) can improve glycemic control and lipid profile. Sevelamer is a calcium free phosphate binder and has postulated to have a role in prevention of absorption of age. The aim of the study was to compare the mean change HbAlc and lipid profile in diabetic kidney disease by selvelamer vs calcium carbonate. The mean of the patient was 58.53 years. Sevelamer showed a mean decrease in HbAlc and lipid profile as compared to calcium carbonate $(-0.57+45,-8.35 \pm 9.006,-1.40 \pm 7.07$, $-4.20 \pm 4.26$, vs $-0.08 \pm 0.21,0.10 \pm 2.51,0.62 \pm 1.03,1.00 \pm 1.55)$. However there was a mean rise in C-reactive protein. We concluded that sevelamer caused greater mean decrease that in HBAlc and lipid profile as compared to calcium carbonate but failed to decrease $C$ - reactive protein.
\end{abstract}

Keywords: Advanced Glycation end Products (AGE), Diabetic Nephropathy, Glomerular Filtration Rate (GFR), Hyperfiltration, Nephrotic Syndrome, End Stage Renal Disease (ESRD), Chronic Kidney Disease (CKD), UK Prospective Diabetic Study (UKPDS), HbA1C, C - reactive protein (CRP), High density Lipoprotein (HDL), Low Density Lipoprotein (LDL), Chronic Renal insufficiency

\section{Introduction}

Diabetic nephropathy $(\mathrm{DN})$ is a clinical syndrome characterized by persistent albuminuria ( $>300 \mathrm{mg} / \mathrm{dl}$ or $>200 \mathrm{mg} / \mathrm{dl}$ ) that is confirmed on at least 2 occasions 3-6 months apart, progressive decline in the glomerular filtration rate (GFR) and elevated arterial blood pressure [1]. This nephropathy has a long natural history in patients with type 1 diabetes. In the beginning, the patient shows hyper filtration, followed by occasional occurrence of micro albuminuria. The patient then shows a gradual a gradual decline of the GFR and persistence of micro albuminuria culminating in mild to moderate proteinuria. The final step of the natural history of the disease is characterized by severe proteinuria with or without nephrotic and chronic renal insufficiency that declines to ESRD [2]. DN is major factor in the development of chronic kidney disease (CKD) and is recognized as the leading cause of end stage renal disease around the world. DN occurs in $15-40 \%$ people with type 1 diabetes, with a peak incidence at 15-20 years disease duration. In type 2 diabetes, the prevalence is $5-20 \%$, with the condition being more common in people of Asian or African descent. In the UKPDS (UK Prospective Diabetes Study), 38 \% developed albuminuria and 29\% developed renal impairment after 15 days of follow up $[3,4]$.

Advanced glycation end products (AGEs) are formed when elevated levels of glucose start forming covalent adducts with plasma proteins through a non-enzymatic process known as glycation [5]. Glycation of proteins leading to AGEs formation is thought to be an important cause of diabetic nephropathy [6]. GE results in imbalance between extracellular matrix formation and degradation, resulting in increased deposition of collagen, fibronectins and laminin. This leads to altered density of collagen as a result of glycation, resulting in altered cell adhesion and cell growth. This results in inhibition of assembly of collagen and laminin [7]. Sevelamer is nonabsorbable, calcium free and albumin free anion exchange resin, which binds to dietary phosphate in the gastrointestinal tract [8]. Previous studies have shown that sevelamer reduces absorption of AGEs present in our food [9,10]. This study was carried out to evaluate this beneficial effect of sevelamer. This study may be useful to ascertain the beneficial effect of sevelamer in reducing $\mathrm{HbA1c}$, serum lipids and inflammatory 
markers in Pakistani population.

\section{Materials and Methods}

This study was carried out at Nephrology Department Akbar niazi teaching hospital Islamabad from 01-11-2017 to 30-042018. Patients with gender, having age $>$ or equal to 30 years and $<$ or equal to 70 years and with type 2 diabetes who were being treated with at least one medication and had diabetic kidney disease as per protocol were included in the study . Patients on treatment for hyperphosphatemia, biopsy proven kidney disease other than diabetic kidney disease, having hypophosphatemia and hypercalcemia were excluded from the study.

Patients fulfilling the inclusion and exclusion criteria were enrolled in the study. An informed consent was taken from patients before including them in the study. Demographics of the patient which include name, age, gender, medical registration number, address and contact number was recorded. Duration of diabetes was also recorded. Baseline $\mathrm{HbAlc}$, lipid profile and $\mathrm{C}$ reactive protein of all the patients were measured. Patients were randomized into two groups by lottery method. One of these groups was given Sevelemer 1600mg thrice daily, while the other group was given calcium carbonate $80 \mathrm{mg}$ thrice daily. $\mathrm{HbA} 1 \mathrm{c}$, lipid profile and $\mathrm{C}$ reactive protein was measured after three months. After three weeks a cross over technique was applied after wash out period of 1 week. Patients receiving Sevelemer were now given calcium carbonate and vice versa. $\mathrm{HbAlc}$, lipid profile and $\mathrm{C}$ reactive protein was again measured after 3 months.

The mean change in HA1c, fasting cholestrol, LDL, triglyceride and $\mathrm{C}$ reactive protein after using sevelamer and calcium carbonate was calculated by using student $t$-test with $\mathrm{p}$ value $<$ or equal to 0.05 as significant. Data was stratified for age, gender and duration of diabetes. Post stratification student $\mathrm{t}$ - test was applied and a p- value $<$ or equal to 0.05 was considered significant.

\section{Resullts}

The mean age of the patients was $58.53+=8.90$ years. $31.1 \%$ $(n=87)$ of the patients had age $30-49$ years, while $68.9 \%(n=$ 1930 of patients had age 50-70 years. Gender distribution shows that $53.2 \%(n=149)$ were males while $46.8 \%(n=131)$ were females. The mean duration of diabetes was 10.45 years. The mean change in $\mathrm{HbA1c}$, total cholesterol, HDL, $\mathrm{LDL}$, triglyceride and $\mathrm{C}$ reactive protein after using calcium carbonate for 3 months $-0.08 \pm 0.21,0.100 .10 \pm 2.51,0.6 \pm$ $6.39,0.62 \pm 1.03,1.00 \pm 1.55$ and $.10 \pm 0.07$ respectively. The mean change in HbAlc, total cholesterol, HDL, LDL, triglyceride and $\mathrm{C}$ reactive protein after using sevelamer for 3 months was $0.57 \pm 0.45,8.35 \pm 9.006,1.79 \pm 10.85,-1.04 \pm$ $7.07,-4.20 \pm 4.26$ and $0.54 \pm .42$ respectively. (Table No. 1) Results were stratified with respect to age with $p$-value $<0.05$ as significant. \pm

\begin{tabular}{|c|c|c|c|c|c|c|}
\hline & \multicolumn{3}{|c|}{ Calcium Carbonate } & \multicolumn{3}{|l|}{ Sevelamer } \\
\hline & Mean change & SD & P-value & Mean change & SD & P-value \\
\hline $\mathrm{HbA} 1 \mathrm{C}$ & -0.08 & 0.21 & 0.000 & -0.575 & 0.45 & 0.000 \\
\hline Total cholesterol mg/dl & 0.10 & 2.51 & 0.506 & -8.35 & 9.006 & 0.000 \\
\hline $\mathrm{HDL} \mathrm{mg} / \mathrm{dl}$ & -0.6 & 6.39 & 0.71 & 1.79 & 10.85 & 0.15 \\
\hline LDL mg/dl & 0.62 & 1.03 & 0.000 & -1.40 & 7.072 & 0.001 \\
\hline Triglyceride $\mathrm{mg} / \mathrm{dl}$ & 1.00 & 1.55 & 0.000 & -4.20 & 4.26 & 0.000 \\
\hline $\mathrm{C}$ reactive proteins $\mathrm{mg} / \mathrm{dl}$ & 0.10 & 0.07 & 0.000 & 0.54 & 0.427 & 0.000 \\
\hline
\end{tabular}

Table 1.0

\begin{tabular}{|l|l|l|l|l|l|l|}
\hline \multirow{2}{*}{} & \multicolumn{4}{|l}{ Calcium Carbonate } & \multicolumn{2}{l|}{ Sevelamer } \\
\cline { 2 - 7 } & Age & $\begin{array}{l}\text { Mean } \\
\text { change }\end{array}$ & P-value & Age & $\begin{array}{l}\text { Mean } \\
\text { change }\end{array}$ & P-value \\
\hline \multirow{3}{*}{ HbA1C } & $30-49$ years & -0.09 & 0.000 & $30-49$ years & -0.64 & 0.000 \\
\hline \multirow{3}{*}{ Total cholesterol } & $50-70$ years & -0.08 & 0.000 & $50-70$ years & -0.54 & 0.000 \\
\hline \multirow{3}{*}{ HDL } & $30-49$ years & 0.13 & 0.609 & $30-49$ years & -8.71 & 0.000 \\
\hline \multirow{3}{*}{ LDL } & $50-70$ years & 0.08 & 0.648 & $50-70$ years & -8.18 & 0.000 \\
\hline \multirow{2}{*}{ Triglyceride } & $30-49$ years & 0.5 & 0.000 & $30-49$ years & 1.7 & 0.000 \\
\cline { 2 - 8 } & $50-70$ years & -0.6 & 0.000 & $50-70$ years & 1.5 & 0.000 \\
\hline & $30-49$ years & 0.60 & 0.000 & $30-49$ years & -1.12 & 0.137 \\
\cline { 2 - 8 } & $50-70$ years & 0.62 & 0.000 & $50-70$ years & -1.52 & 0.003 \\
\hline & $30-49$ years & 1.00 & 0.000 & $30-49$ years & -3.77 & 0.000 \\
\hline & $50-70$ years & 1.00 & 0.000 & $50-70$ years & -4.39 & 0.000 \\
\hline
\end{tabular}




\begin{tabular}{|l|l|l|l|l|l|l|}
\hline \multirow{2}{*}{ C reactive protein } & $30-49$ years & 0.10 & 0.000 & $30-49$ years & 0.50 & 0.000 \\
\cline { 2 - 7 } & $50-70$ years & 0.10 & 0.000 & $50-70$ years & 0.55 & 0.000 \\
\hline
\end{tabular}

Table 2.0

\section{Discussion}

This randomized control trial was conducted to compare the mean change in $\mathrm{HbA} 1 \mathrm{c}$, lipid profile and $\mathrm{C}$ reactive protein by sevelamer as compared to calcium carbonate. Sevelamer is considered to have an emergency anti-inflammatory effect in addition to its role in preventing AGE absorption from the gut. This thought to be due to the resin like property of the drug enabling it to prevent the harmful absorption of AGE from the gut [11]. Avoidance of AGE in food has a documented role in improving of glycemic control as well as preventing diabetic nephropathy [12].

In our study the patients were more or less likely disturbed with respect to gender. Majority of the patients $(68.9 \%)$ were having age 50-70 years. This depicts the burden of diabetes in advancing age. The mean age was 58.3 years. The mean duration was 10.45 years. This depicts that the patients enrolled in the study had long duration of diabetes. The mean change in HbA1c, total cholesterol, HDL, LDL, triglyceride and $\mathrm{C}$ reactive protein after using calcium carbonate for 3 months shows that there was a mean decrease in HbAlc of $0.08 \%$ which was statistically significant. But on the other hand there was a mean increase in total cholesterol, HDL, $\mathrm{LDL}$, triglyceride and $\mathrm{C}$ reactive protein. The mean change in $\mathrm{HbA1c}$, total cholesterol, HDL, LDL, triglyceride and $\mathrm{C}$ reactive protein after using sevelamer for 3 months shows that the mean decrease in $\mathrm{HbAlc}$ by sevelamer was greater as compare to calcium carbonate. It deceased by $-0.57 \%$. Moreover, there was substantial decrease total cholesterol and triglyceride by sevelamer.

After startification for age it was seen that there was greater decease in HbA1c in patients aged 30-49 years as compared to $50-70$ years after using sevelamer ( -0.64 vs 0.54$)$. Similarly there was a greater decrease in total cholesterol in the younger age group . Our results are in concert with the previous studies 9, 10. Sevelamer showed significant reduction in $\mathrm{HbA1c}$, total cholesterol, HDL, LDL, and triglyceride. Although sevelamer is thought have anti-inflammatory action, it is not depicted in our study. There was a mean increase in $\mathrm{C}$ reactive protein after using sevelamer. Further studies need to be done to evaluate the anti-inflammatory effect of sevelamer in our population.

\section{Conclusion}

We conclude that sevelamer causer greater mean decrease in $\mathrm{HbAlc}$, total cholesterol, HDL, LDL, and triglyceride as compared to calcium carbonate. However, sevelamer failed to decrease $\mathrm{C}$ reactive protein.

\section{References}

1. Hadjadi S, Cariou B , Fumeron F, Gand E, Charpentier G , Roussel R, Kasmi AA, Gautier JF, Mohammedi K, Gautier JF, Mohammedi K, Gourdy P, Saulnier PJ (2016) Death, end stage renal disease and renal function decline in patients with diabetic nephropathy in French cohorots of type 1 and type 2 diabets. Diabetologia 59(1): 208-216.

2. pugliease $\mathrm{G}$ (2014) Updating the natural history of diabetic nephropathy . Acta diabetologica 51(6): 905 -915.

3. Bennett K, Aditya BS (2015) An overview of diabetic nephropathy: epidemiology, pathophysiology and treatment. Journal of Diabetes Nursing 19(2): 61-57.

4. Gubitosi-Klug RA, DCCT/EDIC research group (2014) The diabetes control and complications trial/epidemiology of diabetes interventions and complications study at 30 years: summery and future directions. Diabetes care 37(1): 44-49.

5. Singh VP, Bali A, Singh N, Jaggi AS (2014) Advcanced glycation end products and diabetic complications. The Korean Journal of Physiology \& Pharmacology 18(1): 1-4.

6. Nowotny K, Jung T, Hohn A, Weber D, Grune T(2014) Advanced glycation end products and diabetic complications. The Korean Journal of Physiology and Pharmacology.

7. Vlassara H, Uribarri (2014) advanced glycation end products (AGE) and diabetes: cause, effect, or both? Current diabetes reports 14(1): 1-0.

8. Vlassara H, Striker GE (2016) Advanced Glycation Endproducts (AGEs), and Chronic Complications in Diabetes. Principles of Diabetes Mellitus 1-22.

9. Striker GE, Yuberro-Serrano E, Poretsky L, Vlassara H (2014) Control of Oral Advanced Glycation Endproducts (AGEs) by Sevelamer Carbonate Improves Glucose Metabolism and Albuminuria in stage 2-4 Diabetic Kidney Disease (DKD). In Diabetes Clinical Care; Genetics and Epidemiology, 0988.

10. Yubero-Serrano EM, Woodward M, Poretsky L, Vlassara H, Striker GE, AGE-less Study Group. (2015) Effects of Sevelamer carbonate on advanced glycation and antioxidant / pro-oxidant status in patients with diabetic kidney disease. Clinical Journal of the American Society of Nephrology CJN- 07750814.

11. Machowska A, Cerrero JJ, Lindholm B, Stenvinkel P (2016) Therapeutics targeting persistent inflammation in chronic kidney disease. Translational Research 167(1): 204-213.

12. Uribarri J, Cai W, Sandu O, Peppa M, Goldberg T, Vlassara $\mathrm{H}$ (2005) Diet derived advanced glycation end products are major contributors to the body $\backslash \mathrm{s}$ AGE pool and induce inflammation in healthy subjects. Annals of the New York Academy of Sciences 1043(1): 461-466.

Copyright: (C2020 Jais Kumar. This is an open-access article distributed under the terms of the Creative Commons Attribution License, which permits unrestricted use, distribution, and reproduction in anymedium, provided the original author and source are credited. 